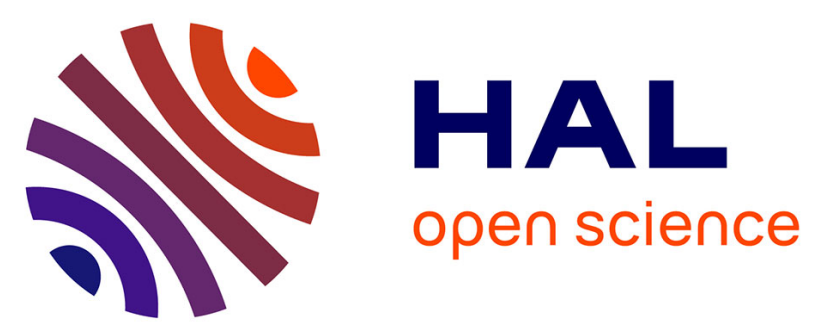

\title{
Real-Time Two-Dimensional Spatiotemporal Terahertz Imaging Based on Noncollinear Free-Space Electrooptic Sampling and Application to Functional Terahertz Imaging of Moving Object
}

Takeshi Yasui, Mukesh Jewariya, Takashi Yasuda, Markus Schirmer, Tsutomu Araki, Emmanuel Abraham

\section{To cite this version:}

Takeshi Yasui, Mukesh Jewariya, Takashi Yasuda, Markus Schirmer, Tsutomu Araki, et al.. Real-Time Two-Dimensional Spatiotemporal Terahertz Imaging Based on Noncollinear Free-Space Electrooptic Sampling and Application to Functional Terahertz Imaging of Moving Object. IEEE Journal of Selected Topics in Quantum Electronics, 2013, 19 (1), pp.8600110. 10.1109/JSTQE.2012.2210393 . hal-00786150

\author{
HAL Id: hal-00786150 \\ https://hal.science/hal-00786150
}

Submitted on 12 Mar 2018

HAL is a multi-disciplinary open access archive for the deposit and dissemination of scientific research documents, whether they are published or not. The documents may come from teaching and research institutions in France or abroad, or from public or private research centers.
L'archive ouverte pluridisciplinaire HAL, est destinée au dépôt et à la diffusion de documents scientifiques de niveau recherche, publiés ou non, émanant des établissements d'enseignement et de recherche français ou étrangers, des laboratoires publics ou privés.

\section{(1)(1) $\$(0)$}

Distributed under a Creative Commons Attribution - NonCommercial - ShareAlikel 4.0 


\title{
Real-Time Two-Dimensional Spatiotemporal Terahertz Imaging Based on Noncollinear Free-Space Electrooptic Sampling and Application to Functional Terahertz Imaging of Moving Object
}

\author{
Takeshi Yasui, Mukesh Jewariya, Takashi Yasuda, Markus Schirmer, Tsutomu Araki, and Emmanuel Abraham
}

\begin{abstract}
We review 2-D spatiotemporal (2D-ST) terahertz (THz) imaging based on noncollinear free-space electrooptic sampling and its application to $\mathrm{THz}$ reflection tomography, $\mathrm{THz}$ spectral imaging, and THz spectral computed tomography (CT). 2D-ST THz imaging enables high-speed image acquisition at much higher rates than previously. Two-dimensional $\mathrm{THz}$ reflection tomography was effective for visualizing the internal structure of a moving paint film. A THz color scanner demonstrated the potential of rapid nondestructive inspection of moving pharmaceutical tablets. A THz spectral CT system using real-time line projection of a THz beam was effectively applied to a continuously rotating object. 2D-ST THz imaging enables functional $\mathbf{T H z}$ imaging of moving objects in various practical applications.
\end{abstract}

Index Terms-Electrooptic effects, imaging, nondestructive testing, spectroscopy, terahertz radiation.

\section{INTRODUCTION}

$\mathbf{I}$ N RECENT years, pulsed terahertz (THz) radiation has attracted much attention as a new tool for nondestructive inspection, quality control, and material characterization [1], [2]. In particular, $\mathrm{THz}$ imaging with pulsed $\mathrm{THz}$ radiation is one of the most innovative tools in this research field because it enables noncontact, nonionizing, and coherent imaging. Following $\mathrm{THz}$ transmission imaging [3], other unique imaging modalities have been reported, including reflection tomography [4], spectral imaging [5], and computed tomography (CT) [6].

This work was supported by Collaborative Research Based on Industrial Demand from the Japan Science and Technology Agency, Grants-in-Aid for Scientific Research (21650111 and 23656265) from the Ministry of Education, Culture, Sports, Science, and Technology of Japan, the Renovation Center of Instruments for Science Education and Technology in Osaka University, and the Tateishi Science and Technology Foundation, Japan.

T. Yasui and M. Jewariya are with the Institute of Technology and Science, The University of Tokushima, Tokushima, Tokushima 770-8506, Japan (e-mail: yasui@me.tokushima-u.ac.jp; jewariya.mukesh@femto.me.tokushima-u.ac. jp).

T. Yasuda was with the Graduate School of Engineering Science, Osaka University, Toyonaka, Osaka 560-8531, Japan. He is now with Hamamatsu Photonics K.K., Hamamatsu 435-8558, Japan (e-mail: yasuda@crl.hpk.co.jp).

M. Schirmer was with the Graduate School of Engineering Science, Osaka University, Toyonaka, Osaka 560-8531, Japan. He is now with RWTH Aachen University, 52062 Aachen, Germany (e-mail: markus.schirmer @rwth-aachen.de).

T. Araki is with the Graduate School of Engineering Science, Osaka University, Toyonaka 560-8531, Japan (e-mail: araki@me.es.osaka-u.ac.jp).

E. Abraham is with the Laboratoire Ondes et Matière d'Aquitaine, Bordeaux 1 University, Talence Cedex 33405, France (e-mail: em.abraham@loma. u-bordeaux1.fr).
In $\mathrm{THz}$ reflection tomography, a cross-sectional image of the sample can be obtained because this technique employs timeof-flight measurement of $\mathrm{THz}$ echo pulses returning from the boundaries between different materials. One advantage of this technique over conventional methods using ultrasound and $\mathrm{X}$ rays is the ability to function as a noncontact and nonionizing probe. This technique has been used to visualize the internal structure of floppy disks [4], pharmaceutical tablet coatings [7], automobile paint [8], [9], and skin cancer [10].

$\mathrm{THz}$ spectral imaging gives a "color" perspective view of a sample in contrast to the conventional "monochrome" inspection achievable with X-rays and ultrasound. The combined use of $\mathrm{THz}$ color images with $\mathrm{THz}$ spectral fingerprints enables material characterization and spatial mapping, and this approach shows promise for screening of cancer tissue [5] and pharmaceutical tablet [7], detecting explosives and illicit drugs [11], [12], and art conservation [13].

$\mathrm{THz} \mathrm{CT}$ is a ubiquitous technique providing 2-D cross section or 3-D volumetric images of objects in a transmission configuration. Reconstruction of them has been performed by analyzing a series of $\mathrm{THz}$ projection images at different projection angles using the filtered back-projection (FBP) algorithm [14]. This imaging modality has been effectively applied to 3-D objects constituted of low-density, opaque, soft materials, such as plastics [6], wood [15], bone [6], and pharmaceutical tablet [16], in which it is difficult to create good image contrast with X-ray CT because $\mathrm{X}$-rays are too penetrating.

The conventional $\mathrm{THz}$ imaging techniques described above face several practical difficulties when implementing them in real-world applications. One difficulty is the requirement for mechanical time-delay scanning for the temporal waveform measurement of the pulsed $\mathrm{THz}$ electric field. When time-delay scanning in THz imaging is performed using a stepping-motordriven translation stage, the image acquisition time is quite long. This is because the sample has to be raster-scanned in the focal spot of the $\mathrm{THz}$ beam whenever temporal waveform measurement of the THz pulse is completed. Consequently, this method has so far only been applied to stationary objects. If this $\mathrm{THz}$ imaging technique could be extended to moving objects, its potential applications in industry would be greatly increased. 
One effective method to reduce the acquisition time of the temporal waveform is to use a fast scanner for the mechanical time delay, such as galvanometric scanner, a piezoelectric stage, or a rotating delay line [17]. However, there are several drawbacks with these mechanical methods, such as mechanical vibrations, spot size variation of the laser light, and/or the issue of a tradeoff between the scanning range and scanning rate. Asynchronous-optical-sampling $\mathrm{THz}$ time-domain spectroscopy (ASOPS-THz-TDS) seems to be a promising method to accomplish rapid time-delay scanning because it involves no mechanical movement [18]-[22]. Time-delay scanning at a rate of $10 \mathrm{kHz}$ has been achieved using a 1-GHz Ti:Sapphire laser [19]. However, signal integration of numerous scans is required due to the fact that a lock-in amplifier cannot be used in this method. Furthermore, mechanical rastering of the sample position is still required because this method is based on the point-by-point measurement.

Rapid image acquisition can be achieved if instead of a mechanical stage, we use an alternative technique for measuring the time delay and sample position simultaneously. One effective method to realize a stage-free configuration is a combination of a single-shot measurement of the temporal waveform and its 1-D transverse imaging, enabling real-time twodimensional spatiotemporal (2D-ST) imaging. In $\mathrm{THz}$ region, there are a few reports on single-shot temporal measurement of $\mathrm{THz}$ pulses requiring no mechanical stage for time delay, all of which are based on two-dimensional free-space electrooptic sampling (2D-FSEOS) [23]. These include time-to-wavelength conversion using a chirped probe pulse [24], use of a $\mathrm{THz}$ streak camera [25], and time-to-space conversion based on noncollinear 2D-FSEOS [26]. Although some of these techniques could be extended to 2D-ST THz imaging [27], noncollinear 2D-FSEOS should prove most suitable since it only requires a slight and inexpensive modification of a regular 2D-FSEOS system. More importantly, it has no limitations on time resolution caused by coupling between frequency- and time-domain methods [28] or by the instrumental response [25]. We have previously proposed a $2 \mathrm{D}-\mathrm{ST} \mathrm{THz}$ imaging technique based on a combination of noncollinear 2D-FSEOS and line focusing of a THz beam onto a sample [29]. This 2D-ST THz imaging technique has achieved, for the first time, functional THz imaging of moving objects in different modalities, such as reflection tomography [29], spectral imaging [30], [31], and spectral CT [32].

In this paper, we review recent work on three functional $\mathrm{THz}$ imaging techniques, based on 2D-ST THz imaging, that are applicable to moving objects. After the principle of $2 \mathrm{D}-\mathrm{ST} \mathrm{THz}$ imaging based on noncollinear 2D-FSEOS is described in Section II, this method is compared with other methods in Section III. Then, real-time 2-D THz reflection tomography and its application to moving paint films are presented in Section IV. In Section V, a THz spectral imaging system using real-time line scanning of the $\mathrm{THz}$ beam and its application to moving pharmaceutical tablets are described. Section VI describes fast THz CT with real-time line projection that is effectively applied to cross-sectional imaging of continuously rotating objects. The paper is summarized in Section VII.

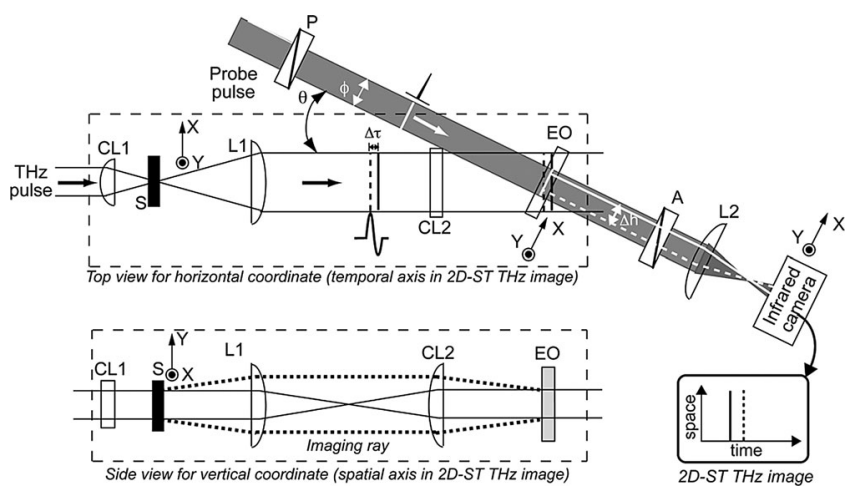

Fig. 1. Typical configuration of 2D-ST THz imaging. (CL1 and CL2: THz cylindrical lenses; L1: THz planoconvex lens; L2: optical planoconvex lens; S: sample; EO: electrooptic crystal; P: polarizer; A: analyzer.)

\section{PRINCIPLE OF 2D-ST THZ IMAGING BASED ON NONCOLLINEAR 2D-FSEOS}

Fig. 1 shows a typical configuration for 2D-ST THz imaging based on noncollinear 2D-FSEOS, using an intense THz pulse generated by an amplified femtosecond laser pulse. A THz cylindrical lens (CL1) is used to produce a line-focused THz beam along the $Y$-axis of the sample (see top and side views in Fig. 1). The line-focused $\mathrm{THz}$ beam on the sample is imaged along the $Y$-axis of an electrooptic (EO) crystal for 2D-FSEOS by using a THz planoconcave lens (L1) and a cylindrical lens (CL2) (see side view in Fig. 1).

On the other hand, as a probe beam, we use another beam that crosses the EO crystal at an angle of $\theta$ with respect to the THz beam. When the temporal width of the probe pulse is much shorter than that of the THz pulse, the wavefront of the $\mathrm{THz}$ beam at different times overlaps with that of the probe beam at different transverse positions. In this case, we use a 2D-FSEOS technique in the noncollinear configuration as a sampling method. This noncollinear 2D-FSEOS method converts the time profile of the pulsed $\mathrm{THz}$ electric field to a spatial polarization distribution of the probe beam [26]. After changing this spatial polarization distribution to a spatial intensity distribution using crossed Nichol prisms (P and A), a 2D-ST $\mathrm{THz}$ image is detected as a probe light image with an infrared camera via an optical imaging lens (L2). The resulting image is composed of the time profile of the $\mathrm{THz}$ pulse and the line image of the sample, which are developed along the horizontal and vertical coordinates of the camera, respectively. Since this 2D-ST THz image is obtained without any mechanical scanning, its acquisition rate can be increased up to the repetition rate of the $\mathrm{THz}$ pulses in principle, for example, $1 \mathrm{kHz}$ in our case.

Here, we assume that the pulsed $\mathrm{THz}$ electric field has a negative peak and a positive peak separated by a relative time delay of $\Delta \tau$ (indicated by the solid and broken line segments in the top view in Fig. 1). The resultant time-to-space conversion factor is given by

$$
\Delta \tau=\frac{(\Delta h \cdot \tan \theta)}{c}
$$




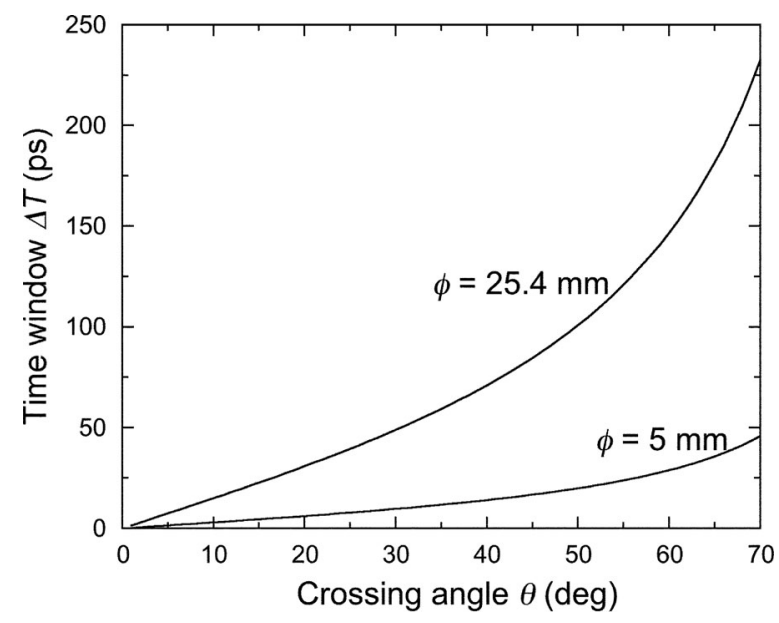

Fig. 2. Relationship between crossing angle $\theta$ and time window $\Delta T$ with respect to different probe beam diameters $\phi$ in noncollinear 2D-FSEOS.

where $\Delta h$ is the spatial distribution of the probe light intensity transcribed from the time delay $\Delta \tau$, and $c$ is the velocity of light in vacuum. The temporal profile of the $\mathrm{THz}$ pulse can then be successively developed along the $X$-axis of the camera. The measurable time window $\Delta T$ is defined as

$$
\Delta T=\frac{(\phi \cdot \tan \theta)}{c}
$$

where $\phi$ is the diameter of the probe beam. Fig. 2 shows the measurable time window $\Delta T$ with respect to different angles $\theta$ in the case of $\phi=5 \mathrm{~mm}$, used in the following 2-D THz reflection tomography, and $25.4 \mathrm{~mm}$, used in the following $\mathrm{THz}$ spectral imaging and spectral CT. In this way, one can set $\Delta T$ arbitrarily by selection of $\theta$ and $\phi$.

\section{COMPARISON With OTHER MEthods}

Here, let us compare our method with other methods for 2DST THz imaging. Although all 2D-ST THz imaging methods are based on 2D-FSEOS [23], they can be categorized into three types based on single-shot measurement method of temporal waveform; time-to-wavelength conversion based on a chirped probe pulse [24], time-to-space conversion using streak camera [25], and time-to-space conversion based on noncollinear 2D-FSEOS [26]. In the first method, by combining a linearly chirped optical probe pulse and a multichannel spectrometer, the temporal waveform of a $\mathrm{THz}$ pulse can be converted into the wavelength spectrum of an optical probe pulse. Such a time-towavelength conversion method can be applied to $2 \mathrm{D}-\mathrm{ST} \mathrm{THz}$ imaging [27]. However, the time resolution in this method is limited by a tradeoff inherent in combining frequency- and timedomain methods [28], though this limitation can be overcome by the noncollinear cross correlation of EO-modulated optical pulses, requiring a more complicated optical setup [33]. Furthermore, use of the spectrometer equipped with narrow entrance slit makes it difficult to introduce the line-imaging configuration into this method. The second method is the use of a long probe pulse and a streak camera. In this method, the temporal profile of the EO modulated probe pulse is transformed into the spatial distribution of the probe light intensity through time-to-space conversion in a streak tube and is then detected with an infrared camera. Although this method may be also extended to 2D-ST THz imaging, the time resolution of this method is limited by the response of the streak camera, typically from subpicosecond to picosecond. Furthermore, this single-shot measurement method needs expensive and complicated streak camera. The third is the use of 2D-FSEOS with the THz beam and optical probe pulse in a noncollinear geometry (noncollinear 2D-FSEOS). In this geometry, direct time-to-space conversion occurs through the spatial overlap of the two noncollinear beams in an EO crystal and the resulting spatial distribution of the probe beam is detected with an infrared camera. This method is easily achieved by a slight modification of a regular 2D-FSEOS system without any expensive apparatus or complicated setup, and most advantageously, it has no limitations on time resolution caused by the intrinsic coupling between frequency- and time-domain methods [28] or the instrumental response [25]. Such simple configuration enables us to easily extend this method to functional $\mathrm{THz}$ imaging such as reflection tomography, spectral imaging, or CT.

It will be also interesting to compare our 2D-ST THz imaging method with ASOPS-THz-TDS method. In ASOPS-THzTDS method, two mode-locked lasers with slightly mismatched mode-locked frequencies are used for generation and detection of the pulsed $\mathrm{THz}$ radiation. This asynchronous optical sampling enables us to linearly expand the time scale of picosecond $\mathrm{THz}$ pulse to microsecond order. The resulting slow signal can be captured rapidly on a standard oscilloscope without the need for time-consuming, mechanical time-delay scanning. Although the rate of time-delay scanning can reach kilohertz order, many scans have to be integrated together to achieve an adequate signal-to-noise ratio (SNR) because a lock-in amplifier cannot be used in this method. Here, we compare 2D-ST THz imaging [30] with ASOPS-THz-TDS [22] from a viewpoint of a relation between SNR of temporal waveform and acquisition time. Fig. 3(a) shows a comparison of SNR between them with respect to acquisition time required for single measurement. Both methods indicated comparable performance within the range of acquisition time from 50 to $50000 \mathrm{~ms}$. However, to compare the performance of them more fairy, we should consider difference of the data acquisition method between them because ASOPS-THz-TDS captures a single temporal waveform of $\mathrm{THz}$ pulse, whereas 2D-ST THz imaging enables parallel measurement of multiple temporal waveforms. Since 2D-ST THz imaging method can capture 232 temporal waveforms of the $\mathrm{THz}$ pulse in parallel during this acquisition time [30], we modified the horizontal scale in Fig. 3(a) to the acquisition time required for 232 temporal waveforms, as shown in Fig. 3(b). It is obvious that 2D-ST THz imaging surpasses ASOPS-THz-TDS in SNR in the case of imaging measurement.

\section{TWO-DIMENSIONAL THZ REFLECTION TOMOGRAPHY OF MOVING OBJECTS}

Fig. 4 shows the experimental setup used for 2-D THz reflection tomography. Amplified femtosecond pulse light 


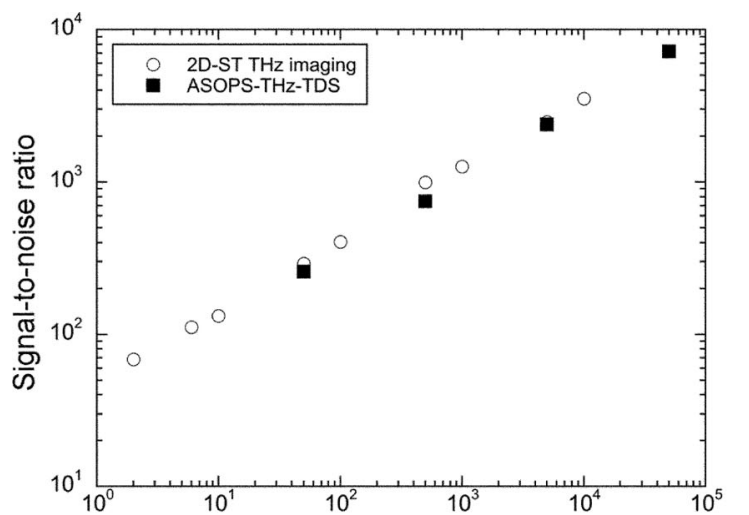

Acquisition time required for single measurement (ms)

(a)

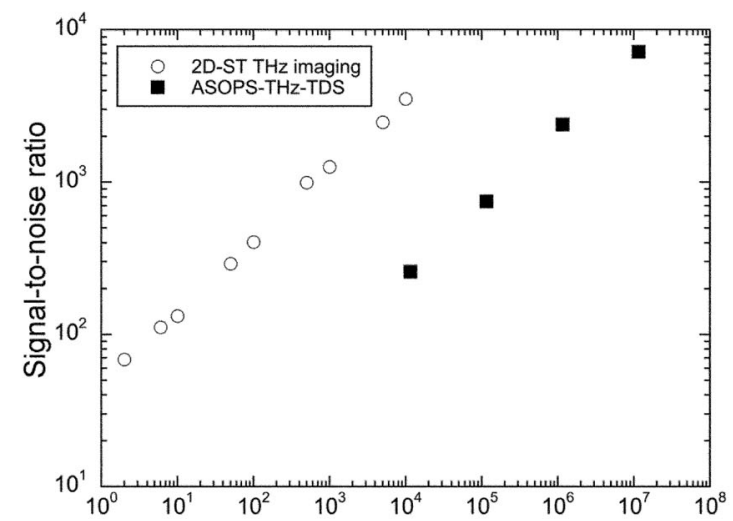

Acquisition time required for 232 temporal waveforms (ms)

(b)

Fig. 3. Comparison of 2D-ST THz imaging with ASOPS-THz-TDS regarding a relation between SNR and acquisition time required for (a) single measurement and (b) 232 temporal waveforms.

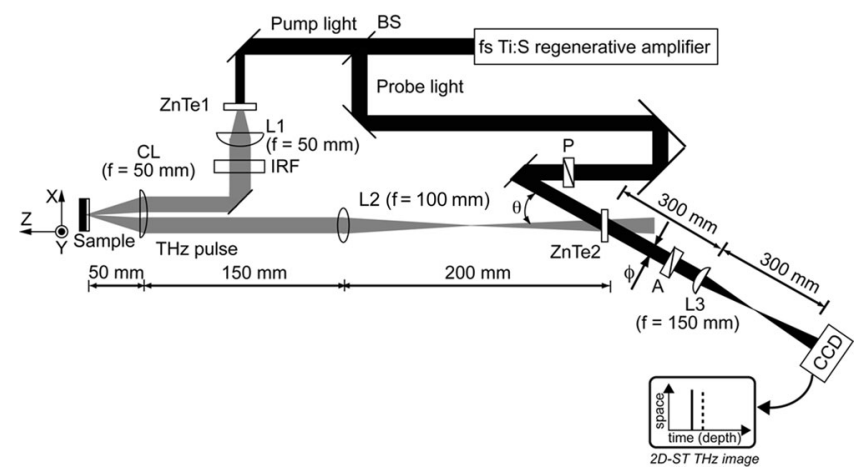

Fig. 4. Experimental setup for 2-D THz reflection tomography. (BS: beam splitter; ZnTe1 and ZnTe2: zinc telluride crystals; L1: THz planoconvex lens; IRF: infrared-cut filter; CL: THz cylindrical lens; L2: THz biconvex lens; P: polarizer; A: analyzer; L3: optical planoconvex lens; CCD: charge-coupleddevice camera.)

$(1 \mathrm{~mJ} /$ pulse, $150 \mathrm{fs}, 800 \mathrm{~nm}, 1 \mathrm{kHz})$ was separated into pump light for $\mathrm{THz}$ generation and probe light for $\mathrm{THz}$ detection. An intense $\mathrm{THz}$ pulse was generated via optical rectification of the pump light in a ZnTe crystal (ZnTe1; $\langle 110\rangle$ orientation, $10 \mathrm{~mm}$ $\times 10 \mathrm{~mm} \times 4 \mathrm{~mm}$ thick). A THz planoconvex lens (L1, $f=$ $50 \mathrm{~mm}$ ) was used to collimate the generated $\mathrm{THz}$ beam. The collimated $\mathrm{THz}$ beam was then delivered to the optical sys-

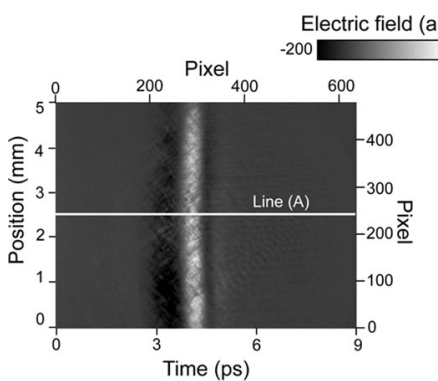

(a)

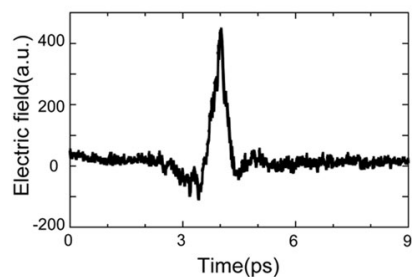

(b)
Fig. 5 (a) 2D-ST THz image of a flat Al mirror surface $\left(\theta=28^{\circ}\right.$, image size $=9 \mathrm{ps} \times 5 \mathrm{~mm}$, and CCD exposure time $=70 \mathrm{~ms}$ ) and (b) temporal waveform of the THz electric field extracted from a line (A) in the 2D-ST THz image.

tem for 2D-ST THz imaging, using a setup slightly modified from that in Fig. 1 for a reflection configuration. A THz cylindrical lens $(\mathrm{CL}, f=50 \mathrm{~mm}$ ) along the $Y$-axis of the sample was used to line-focus the $\mathrm{THz}$ beam onto the sample. The reflected THz beam from the sample was imaged on another ZnTe crystal for noncollinear 2D-FSEOS (ZnTe2; $\langle 110\rangle$ orientation, $10 \mathrm{~mm} \times 10 \mathrm{~mm} \times 1 \mathrm{~mm}$ thick) by $\mathrm{CL}$ and a $\mathrm{THz}$ biconvex lens (L2, $f=100 \mathrm{~mm}$ ). The probe beam, with a diameter of $5 \mathrm{~mm}$, was incident on the crystal ZnTe2 noncollinearly with the THz beam at a crossing angle $\theta$. A 2D-ST THz image was obtained using crossed Nichol prisms ( $\mathrm{P}$ and $\mathrm{A}$ ) near the zero optical transmission point on the EO crystal [34]. Finally, the 2D spatial distribution of the probe light intensity, corresponding to the 2D-ST THz image, was imaged via a planoconvex lens (L3, $f=150 \mathrm{~mm}$ ) onto a 12-bit thermoelectric-cooled chargecoupled-device $(C C D)$ camera $(640 \times 480$ pixels, 10 frames/s). The resulting 2D-ST THz image reflected a $Y Z$ cross-sectional image of the sample because the horizontal coordinate of the 2D-ST THz image corresponded to the time delay of the THz echo pulse, and hence the depth profile of the sample.

A single-frame 2D-ST THz image of a flat aluminum mirror surface is shown in Fig. 5(a) (image size $=9 \mathrm{ps} \times 5 \mathrm{~mm}, \theta$ $=28^{\circ}$ ). The white and black areas in the image indicate positive and negative $\mathrm{THz}$ electric field, respectively. The vertical white line around $4.1 \mathrm{ps}$ in Fig. 5(a) indicates the flat surface of the mirror without any internal structure. Fig. 5(b) shows the temporal waveform of the pulsed $\mathrm{THz}$ electric field extracted from a line profile of the 2D-ST THz image [see line (A) in Fig. 5(a)], in which a pulse duration of $0.5 \mathrm{ps}$ and SNR of 80 were achieved.

$\mathrm{THz}$ paintmeters for automobile paint are an interesting application of THz reflection tomography [8], [9], [29]. We adapted our system for this application and evaluated its performance as a real-time $\mathrm{THz}$ paintmeter. A film of white alkyd paint (group refractive index in $\mathrm{THz}$ region $n_{g}=2.14$ ) was applied to half of the surface of an $\mathrm{Al}$ substrate and allowed to dry (see upper part of Fig. 6). While the sample was continuously moved parallel to the $Y$-axis in Figs. 4 and 6 (moving speed $=5 \mathrm{~mm} / \mathrm{s}$ ), 2-D cross-sectional images of the moving paint film were successively captured at a frame rate of 10 frames/s. The lower part of Fig. 6 shows the cross-sectional images of the sample at three 


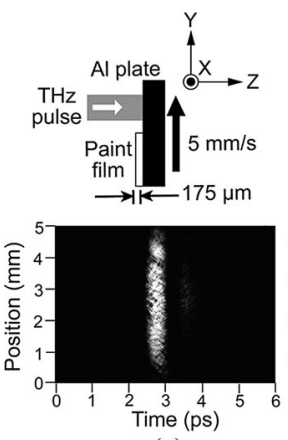

(a)

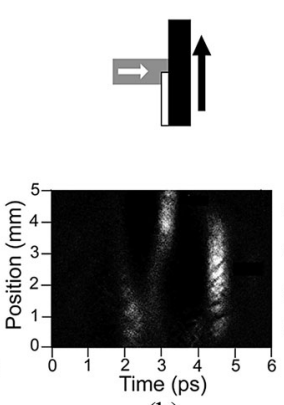

(b)

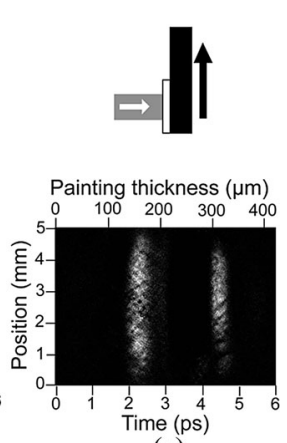

(c)
Fig. 6. Two-dimensional THz tomography at three different illuminating positions of a moving paint film covering half of a substrate $\left(\theta=20^{\circ}\right.$, image size $=6 \mathrm{ps} \times 5 \mathrm{~mm}$ ): (a) unpainted area, (b) boundary between unpainted and painted areas, and (c) painted area.

different illuminating positions (image size $=6 \mathrm{ps} \times 5 \mathrm{~mm}, \theta=$ $20^{\circ}$ ). The unpainted area in Fig. 6(a) shows only one $\mathrm{THz}$ echo signal due to the absence of internal structures. At the boundary between the unpainted and painted areas in Fig. 6(b), although a mixture of one and two echoes should be observed, insufficient transverse resolution blurred the edge at the boundary between the two areas. In the case of the painted area in Fig. 6(c), two $\mathrm{THz}$ echo signals were observed at 2.3 and 4.5 ps from the airpaint boundary and the paint-substrate boundary. The thickness of the paint film $d$ can be obtained using the time separation $\Delta \tau$ between the two echoes and the group refractive index $n_{g}$ of the paint film as follows:

$$
d=\frac{(c \Delta \tau)}{\left(2 n_{g}\right)}
$$

The actual dimensions of this paint film are given on the upper horizontal axis in Fig. 6(c). From this experiment, we confirmed that this paint film had an uneven thickness of $162 \pm 21 \mu \mathrm{m}$ $[$ (mean) \pm (standard deviation) $]$ along the $Y$-axis of the sample. Since the paint thickness around the center of the painted area was determined to be $175 \mu \mathrm{m}$ by a contact-type thickness meter (precision $=3 \%$ ), both methods were reasonably consistent with each other. The thickness resolution $d_{\mathrm{min}}$ in the present system was determined from the duration of the THz pulse $(\Delta t)$ and the group refractive index of the sample, $n_{g}$, as follows:

$$
\begin{aligned}
d_{\text {min }} & =(c \Delta t) /\left(2 n_{g}\right) \\
& =\left(3 \times 10^{8} \times 0.5 \times 10^{-12}\right) /(2 \times 2.14)=35 \mu \mathrm{m} .
\end{aligned}
$$

One interesting application of real-time 2-D reflection tomography will be in the field of art conservation as well as car painting. In this field, it is required to know history of the restoration of an old painting without destructive sampling. Recently, THz reflection tomography has attracted attention in this field because it nondestructively provides the cross-sectional image of the overlapping paint layer based on the optical thickness [13]. However, existing THz tomography system used for the art conservation needs mechanical scanning of time delay and raster scanning of sample position, resulting in long acquisition time for 2-D cross-sectional image, typically several to

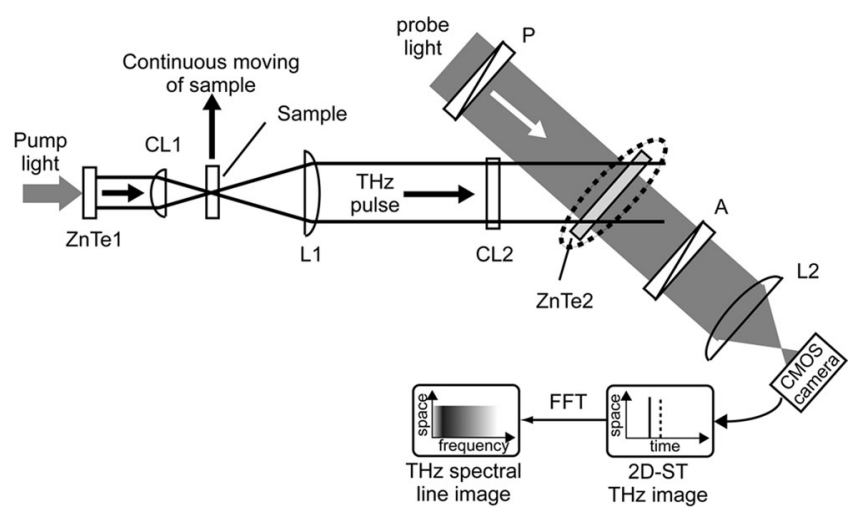

Fig. 7. Experimental setup for THz spectral imaging. (ZnTe1 and ZnTe2: zinc telluride crystals; CL1 and CL2: THz cylindrical lenses; L1: THz planoconvex lens; P: polarizer; A: analyzer; L2: optical planoconvex lens.)

several tens minute. On the other hand, our system can provide the 2-D cross-sectional image in real time and even 3-D volume of its internal structure rapidly. This will lead to the efficient work in the restoration of the old painting.

\section{THz SPectral IMAging of Moving OBJects}

Fig. 7 illustrates the experimental setup used for $\mathrm{THz}$ spectral imaging. Amplified femtosecond pulsed light $(550 \mu \mathrm{J} /$ pulse, $150 \mathrm{fs}, 800 \mathrm{~nm}, 1 \mathrm{kHz}$ ) was used to generate an intense THz pulse via optical rectification in a ZnTe crystal $(\mathrm{ZnTe} 1 ;\langle 110\rangle$ orientation, $25 \mathrm{~mm} \times 25 \mathrm{~mm} \times 1 \mathrm{~mm}$ thick). The generated THz pulse passing through the sample and the probe light from the same laser were made to cross in another ZnTe crystal $(\mathrm{ZnTe} 2 ;\langle 110\rangle$ orientation, $25 \mathrm{~mm} \times 25 \mathrm{~mm} \times 1 \mathrm{~mm}$ thick) for $2 \mathrm{D}-\mathrm{ST} \mathrm{THz}$ imaging. The resulting 2-D image of the probe light, corresponding to the 2D-ST THz image, was imaged with a lens (L2) onto a high-speed 12-bit CMOS camera $(232 \times 232$ pixels, maximum frame rate $=1000$ frames/s) synchronized with the $1-\mathrm{kHz}$ laser pulse. The CMOS camera acquired a 2D-ST THz image of the sample in a dynamic subtraction mode at a frame rate of $500 \mathrm{~Hz}$ [35]. Fourier transformation of the horizontal axis in the 2D-ST $\mathrm{THz}$ image resulted in a $\mathrm{THz}$ spectral line image of the sample along the $\mathrm{THz}$ focal line. To obtain a 2-D spectral image of the sample, the sample was continuously moved across the $\mathrm{THz}$ focal line by a mechanical stage while acquiring a series of line images consecutively (acquisition interval $=100 \mu \mathrm{m}$ ), and the individual line images were then pieced together. Since construction of a 2-D color image by successive stacking of real-time color line image is similar to the processing performed in color scanners, we call this system a THz color scanner [30], [31].

Without any sample, we acquired a $\mathrm{THz}$ spectral line image of the incident $\mathrm{THz}$ power at an image acquisition time of $100 \mathrm{~ms}$, as shown in Fig. 8(a). The horizontal and vertical axes in this image, respectively, show the frequency scale and the spatial scale (frequency window $=2.96 \mathrm{THz}$, frequency resolution $=25.5 \mathrm{GHz}$, spatial window $=20 \mathrm{~mm}$ ). We calibrated the frequency scale of this image using a metal hole array serving as a frequency reference [30]. The power spectrum of the pulsed THz radiation in Fig. 8(b) was extracted along line A in 


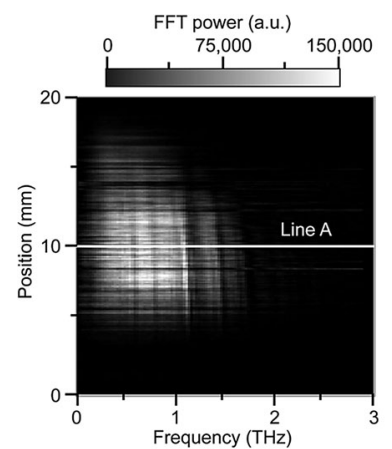

(a)

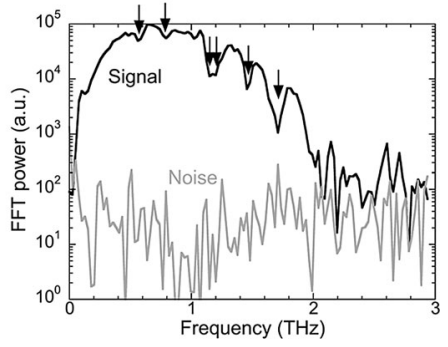

(b)
Fig. 8. Spectral characteristics of $\mathrm{THz}$ profile in the absence of a sample. (a) $\mathrm{THz}$ spectral line images of power and (b) power spectrum along line A (image acquisition rate $=10$ line/s).

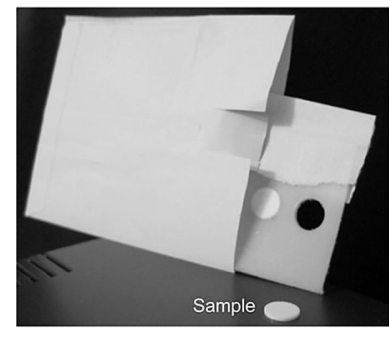

(a)

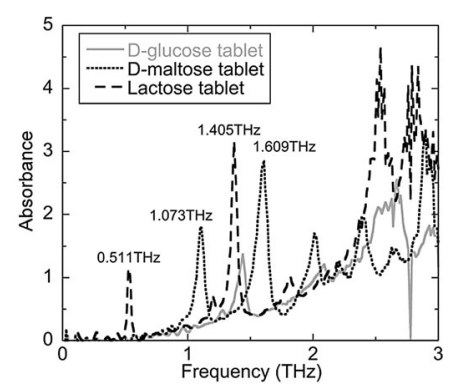

(b)
Fig. 9. (a) Photograph of tablet samples and sample holder concealed in a paper envelope. (b) Absorption spectra of D-glucose, D-maltose, and lactose tablets.

Fig. 8(a). Several absorption lines of atmospheric water vapor were observed (see arrows) because the $\mathrm{THz}$ beam path was not purged with nitrogen gas. To estimate the dynamic range of the $\mathrm{THz}$ signal, we compared the power spectrum with one measured in the absence of the $\mathrm{THz}$ beam (noise spectrum), as shown by the gray line in Fig. 8(b). The SNR of the THz signal was greatly enhanced due to the dynamic subtraction mode of the CMOS camera operating at a frame rate of $500 \mathrm{~Hz}$.

Quality control of pharmaceutical tablets is one promising application of $\mathrm{THz}$ spectral imaging because many medicines show sharp spectral fingerprints in the $\mathrm{THz}$ region resulting from their crystal structures. Although many researches have been performed extensively in this field, achieved acquisition time in existing THz-TDS systems is no more than a few tens $\mathrm{ms}$ at a single-point measurement [7]. This data acquisition rate is still insufficient to perform $\mathrm{THz}$ spectral imaging of moving objects. As a result, the scope of this application does not include total inspection in a manufacturing process. Here, we demonstrate the potential of our system for rapid nondestructive classification of pharmaceutical tablets moving on a translation stage. In this demonstration, we used three different kinds of sugar tablets (D-glucose, D-maltose, and lactose powders mixed with polyethylene powder; diameter $=10 \mathrm{~mm}$, thickness $=$ $1 \mathrm{~mm}$ ) and one nonsugar tablet (polyethylene powder; diameter $=10 \mathrm{~mm}$, thickness $=1 \mathrm{~mm}$ ) as samples, as shown in Fig. 9(a). $\mathrm{THz}$ spectral fingerprints of the sugar tablets were measured beforehand with a commercial THz-TDS system (Otsuka Elec-

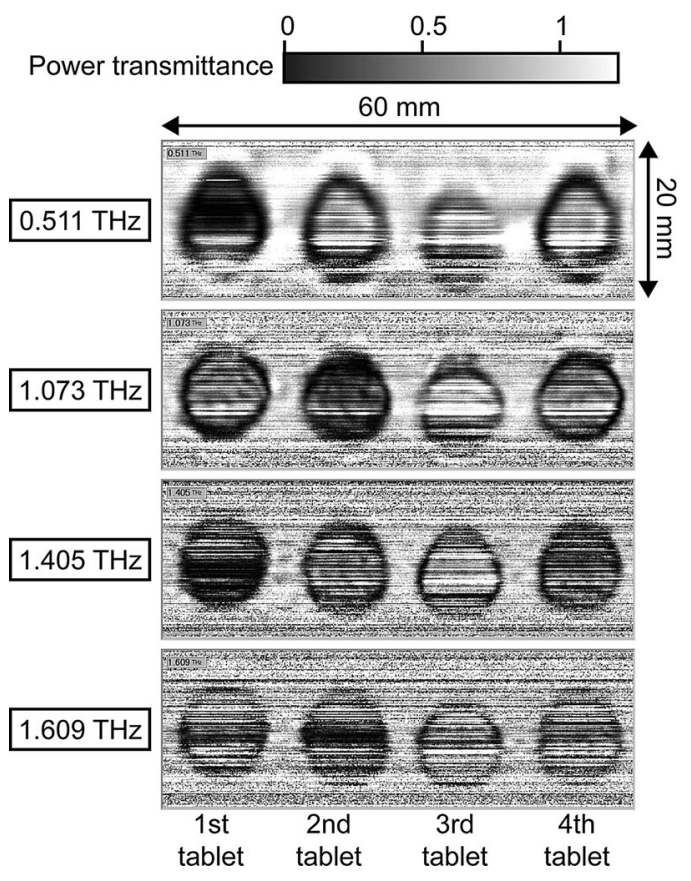

Fig. 10. THz spectral images of transmittance in four tablet samples. Each image consists of $232 \times 600$ pixels (image acquisition time $=60 \mathrm{~s}$ ).

tronics Co., Ltd., TR-1000). Fig. 9(b) compares the absorption spectra among the three sugar tablets. Because characteristic spectral fingerprints appeared in these spectra, it should be possible to classify these sugar tablets based on their $\mathrm{THz}$ spectral fingerprints even though they have a similar appearance.

We acquired 116 consecutive spectral images of the $\mathrm{THz}$ transmittance of the sample in a range from 0 to $2.98 \mathrm{THz}$, with a frequency resolution of $25.5 \mathrm{GHz}$. Fig. 10 shows THz power transmittance images of the samples (image area $=60 \mathrm{~mm} \times$ $20 \mathrm{~mm}$, pixel size $=600$ pixels $\times 232$ pixels, image acquisition time $=60 \mathrm{~s}$ ) at four different frequencies appearing in the $\mathrm{THz}$ spectral fingerprints: $0.511 \mathrm{THz}$ for the lactose, $1.073 \mathrm{THz}$ for the D-maltose, $1.405 \mathrm{THz}$ for the D-glucose and lactose, and $1.609 \mathrm{THz}$ for the D-maltose [see Fig. 9(b)]. The black color in these images corresponds to a low transmittance and therefore to a high absorption. Low-transmittance images appeared at 0.511 and $1.405 \mathrm{THz}$ for the first tablet, at 1.073 and $1.609 \mathrm{THz}$ for the second tablet, and at $1.405 \mathrm{THz}$ for the fourth tablet, respectively. The third tablet did not show low-transmittance images at any frequencies. From these results, we determined that the first, second, third, and fourth samples were the lactose, D-maltose, reference, and D-glucose tablets, respectively.

\section{THz SPECTRAL CT FOR CONTINUOUSLY ROTATING OBJECTS}

To reconstruct 2-D spectral cross section of an object based on CT algorithm, one has to acquire 3-D information composed of one spatial dimension (line image), one color dimension (spectral data), and one angular dimension (projection angle). However, since existing THz CT system is based on both pointby-point and angle-by-angle measurements of the time-domain 


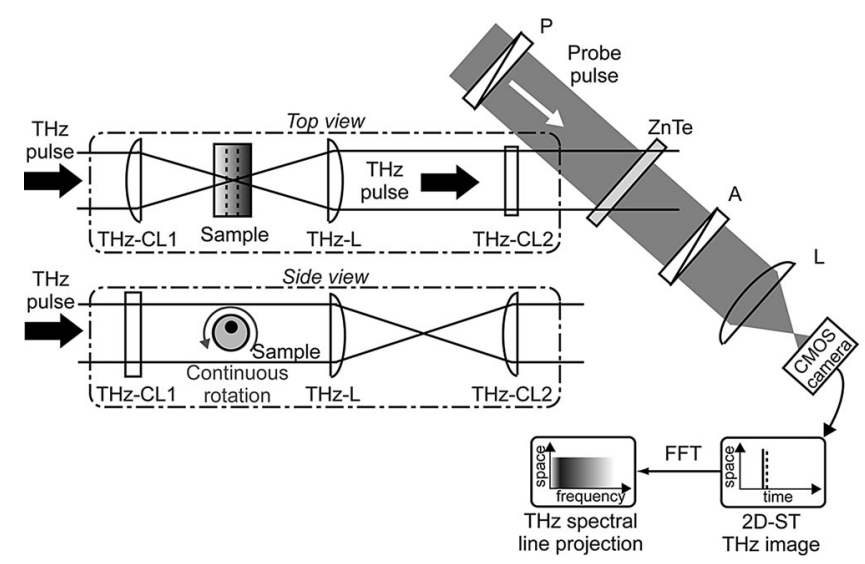

Fig. 11. Experimental setup. (THz-CL1 and THz-CL2: THz cylindrical lenses, THz-L: THz planoconvex lens, P: polarizer, A: analyzer, L: optical planoconvex lens.)

spectrometer, it needs to perform a serial mechanical scanning of time-delay, sample position, and projection angle. The resulting long acquisition time is a strong limitation in practical use. On the other hand, 2D-ST THz imaging provides 2-D information of time delay and sample position simultaneously and hence $\mathrm{THz}$ color line image in real time without the need for mechanical scanning (see Section V). Therefore, if the 2D-ST $\mathrm{THz}$ imaging is applied for $\mathrm{THz} \mathrm{CT}$, only continuous rotation of an object is required for 2-D spectral cross section. This leads to large decrease of acquisition time in $\mathrm{THz}$ spectral CT.

The experimental setup of our $\mathrm{THz}$ spectral CT system is shown in Fig. 11, in which we modified two parts of the $\mathrm{THz}$ color scanner system (see Fig. 7) for THz CT. The first modification is in the optical system for generating an intense $\mathrm{THz}$ pulse (not shown in Fig. 11). To greatly increase the THz pulse energy and hence decrease the image acquisition time, we used Cherenkov radiation from a $\mathrm{LiNbO}_{3}$ crystal excited by a pulsefront-tilted pump beam [36], [37]. We further modified the line focusing of the THz beam across the cross section of the sample by using a THz cylindrical lens (CL1). These modifications enabled us to achieve real-time line projection of the $\mathrm{THz}$ beam on the sample. Then, the THz beam passing through the sample was used for 2D-ST THz imaging. The resulting 2D-ST THz image had a temporal range of $37 \mathrm{ps}$ and a spatial range of $15 \mathrm{~mm}$. Fourier transformation of the time axis in the 2D-ST THz image gave spectral line projection data of the sample (spectral resolution $=27 \mathrm{GHz}$ ).

In the following demonstrations, we rotated the sample through a full turn by continuous rotation for $6 \mathrm{~s}$. During this rotation, 600 line-projection datasets of a 2D-ST THz image were collected at a rate of $100 \mathrm{~Hz}$, resulting in an angular sampling resolution of only $0.6^{\circ}$. In the traditional THz-CT, only 18 (angular step of $10^{\circ}$ ) or 36 (angular step of $5^{\circ}$ ) projections angles are selected owing to the long acquisition time of the pointby-point and angle-by-angle scanning measurement (more than $1 \mathrm{~h}$ ). Therefore, a significant advantage of the proposed system over conventional systems is the ability to perform fast and precise CT imaging. Finally, the spectral cross section of the sample across the THz focal line was reconstructed by analyz-

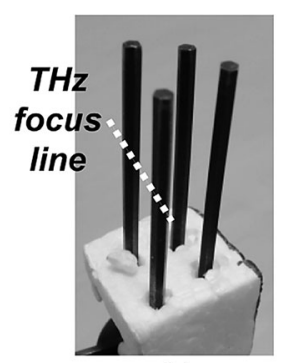

(a)

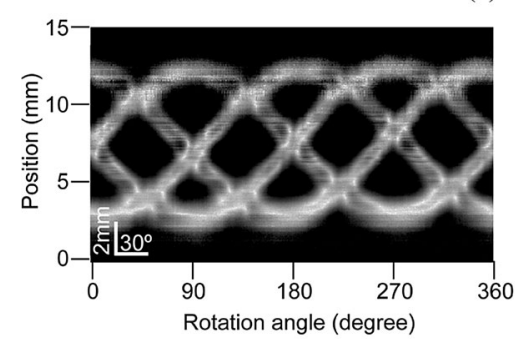

(b)

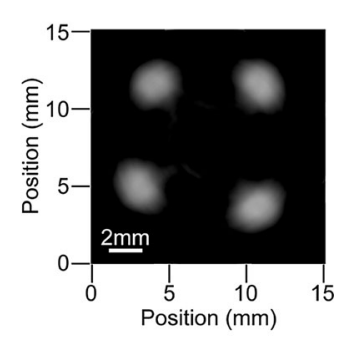

(c)
Fig. 12. Four metallic bars (2-mm diameter). (a) Optical photograph, (b) sinogram of spectral amplitude at $0.6 \mathrm{THz}$, and (c) spectral cross section at $0.6 \mathrm{THz}$.

ing a spectral line projection data series, that is, a sinogram, with the FBP algorithm [14].

We investigated a simple sample consisting of four metallic bars (2-mm diameter) illuminated by a THz focal line, to demonstrate the rapid cross-sectional reconstruction, as shown in Fig. 12(a). Fig. 12(b) shows the sinogram of the spectral amplitude at $0.6 \mathrm{THz}$ for this sample, where the horizontal and vertical coordinates, respectively, give the rotation angle (600 points from $0^{\circ}$ to $360^{\circ}$ ) and the THz projection-line image ( 232 points from 0 to $15 \mathrm{~mm}$ ). The white color indicates a decrease in the spectral amplitude. An isolated distribution of the four metallic bars generated four overlapping sinusoidal waveforms in the sinogram with different phases of the rotation angle. Using the standard FBP algorithm [14], we reconstructed a cross-sectional image corresponding to the portion of the object illuminated by the THz line, as shown in Fig. 12(c). Since white and black colors indicated weak and strong signals in the $\mathrm{THz}$ spectral amplitude, respectively, the white regions in Fig. 12(c) visualize some structures where the signal is decreased. Although no spectroscopic information could be obtained from this metallic sample, the positions and shapes of the four metallic bars were clearly visualized, demonstrating the ability of the system to properly reconstruct cross-sectional images of simple samples in only $6 \mathrm{~s}$.

The second sample was a foam cylinder (16-mm diameter) in which an off-axis cylindrical hole (4-mm diameter) was drilled, as shown in Fig. 13(a). To demonstrate THz spectral CT, the hole was filled with lactose powder mixed with polyethylene powder (30\% lactose and 70\% polyethylene). As shown in Fig. 9(b), lactose exhibits a THz spectral fingerprint at $0.511 \mathrm{THz}$. Fig. 13(b) and (c) represents the sinogram of the spectral amplitude at $0.53 \mathrm{THz}$ and the corresponding reconstructed spectral cross section of the sample, respectively. The area of the foam cylinder was not visible owing to the low refractive index change 


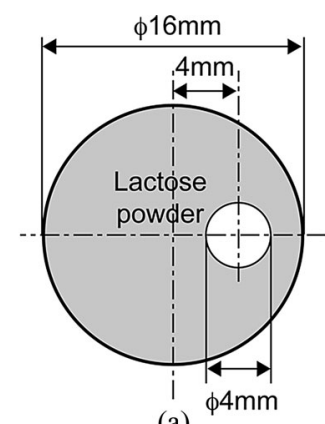

(a)

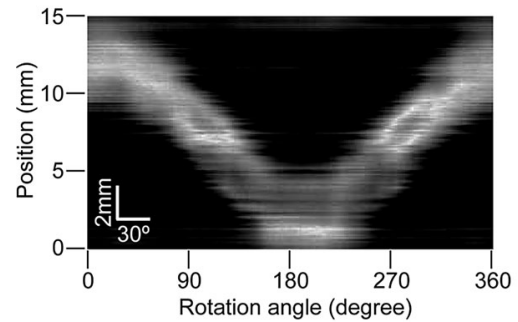

(b)

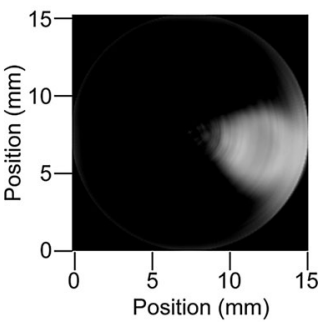

(c)
Fig. 13. Lactose powder filled in one off-axis cylindrical hole formed in foam cylinder. (a) Schematic drawing, (b) sinogram of spectral amplitude at $0.53 \mathrm{THz}$, and (c) spectral cross section at $0.53 \mathrm{THz}$.

from air to foam and the low absorption coefficient of the foam (Rohacell, $\alpha=0.1 \mathrm{~cm}^{-1}$, refractive index $=1.15$ ). In Fig. 13(b) and (c), the position and the size of the cylindrical hole are revealed due to the different transmission of the $\mathrm{THz}$ radiation in this part of the sample. The lower transmission was due to both refraction losses and lactose absorption at $0.53 \mathrm{THz}$.

Distortions of the cross-sectional $\mathrm{THz}$ images were observed in Figs. 12(c) and 13(c). To explain it, we have to take into account several phenomena. First, naturally, diffraction effects are important since the millimeter size object is close to the imaging wavelength. These effects uniformly blurred the final reconstruction of the $\mathrm{THz}$ cross section. However, they cannot explain why the distortion is mainly elliptical concerning the imaging of cylindrical metal bars [see Fig. 12(c)] or lactose powder [see Fig. 13(c)]. Another reason is the position of the cylinder (metallic bar or lactose powder), at the edge of the reconstruction area. For instance, in Fig. 13(b) (sinogram), we can observe that the position of the lactose cylinder is near the edge of the observing area for the angles $0^{\circ}, 180^{\circ}$, and $360^{\circ}$. Consequently, the boundary of the 16-mm diameter foam holder is not directly involved here. During the application of the FBP algorithm, this induces a distortion since the algorithm requires a slightly larger visualization of the cylinder (the cylinder itself and a few mm around) in order to properly determine its actual shape.

\section{CONCLUSION}

We reviewed three techniques for rapid, functional $\mathrm{THz}$ imaging based on 2D-ST THz imaging. It is well known that real-time movie of a moving object can be measured at the fixed timedelay [38]. However, THz imaging without time-delay scanning merely gives monochromatic shadowgraph without any spectral information (or depth information in the case of $\mathrm{THz}$ tomography) in the same manner as X-ray or ultrasound methods. It should be emphasized in this review article that incomparable high-speed data acquisition achieved by 2D-ST THz imaging opens the door to $\mathrm{THz}$ spectral imaging and tomographic imaging of moving objects.

First, using 2-D THz reflection tomography, the temporal evolution of 2-D cross-sectional images was clearly visualized as the illuminating position of the $\mathrm{THz}$ beam moved across a paint sample. Interesting applications of this real-time tomography will be in the field of art conservation as well as car painting. Second, we demonstrated the ability to distinguish and spatially image three different sugar tablets by using a THz color scanner employing real-time line scanning of a $\mathrm{THz}$ beam. This demonstration showed the potential of the $\mathrm{THz}$ color scanner in total inspection for rapid quality control of pharmaceutical tablets moving on a conveyor belt. Third, we showed the potential of real-time $\mathrm{THz}$ line projection for $\mathrm{THz}$ spectral $\mathrm{CT}$ of continuously rotating objects. Owing to the 10-ms acquisition time of the 2D-ST THz image, line projection data were recorded in real time in association with the continuous rotation of the sample.

To the best of our knowledge, this is the first time that these functional $\mathrm{THz}$ imaging techniques have been applied to moving objects. These rapid, functional $\mathrm{THz}$ imaging techniques based on 2D-ST THz imaging will be a powerful tool for sensing, nondestructive inspection, and material characterization of moving objects in practical applications.

\section{ACKNOWLEDGMENT}

The authors would like to acknowledge Dr. K. Kitagishi and Dr. Y. Izutani of Otsuka Electronics Co. Ltd., Japan, for preparation of the sugar tablets and for conducting THz-TDS measurements. They also thank K. Sawanaka, A. Ihara, M. Minami, M. Fujio, and Y. Ohgi of the Graduate School of Engineering Science, Osaka University for their experimental support in part of this work.

\section{REFERENCES}

[1] D. M. Mittleman, Sensing With $\mathrm{THz}$ Radiation. Berlin, Germany: Springer, 2003

[2] M. Tonouchi, "Cutting-edge terahertz technology," Nat. Photon., vol. 1, no. 2, pp. 97-105, Feb. 2007

[3] B. B. Hu and M. C. Nuss, "Imaging with terahertz waves," Opt. Lett., vol. 20, no. 16, pp. 1716-1718, Aug. 1995.

[4] D. M. Mittleman, S. Hunsche, L. Boivin, and M. C. Nuss, "T-ray tomography," Opt. Lett., vol. 22, no. 12, pp. 904-906, Jun. 1997.

[5] T. Löffler, T. Bauer, K. Siebert, H. Roskos, A. Fitzgerald, and S. Czasch, "Terahertz dark-field imaging of biomedical tissue," Opt. Exp., vol. 9, no. 12, pp. 616-621, Dec. 2001.

[6] B. Ferguson, S. Wang, D. Gray, D. Abbott, and X.-C. Zhang, "T-ray computed tomography," Opt. Lett., vol. 27, no. 15, pp. 1312-1314, Aug. 2002.

[7] Y.-C. Shen and P. F. Taday, "Development and application of terahertz pulsed imaging for nondestructive inspection of pharmaceutical tablet," IEEE J. Sel. Topics Quantum Electron., vol. 14, no. 2, pp. 407-415, Mar. 2008.

[8] T. Yasui, T. Yasuda, K. Sawanaka, and T. Araki, "A terahertz paintmeter for non-contact monitoring of thickness and drying progress in paint film," Appl. Opt., vol. 44, no. 32, pp. 6849-6856, Nov. 2005.

[9] T. Yasuda, T. Iwata, T. Araki, and T. Yasui, "Improvement of minimum paint film thickness for THz paint meters by multiple-regression analysis," Appl. Opt., vol. 46, no. 30, pp. 7518-7526, Oct. 2007. 
[10] R. M. Woodward, B. E. Cole, V. P. Wallace, R. J. Pye, D. D. Arnone, E. H. Linfield, and M. Pepper, "Terahertz pulse imaging in reflection geometry of human skin cancer and skin tissue," Phys. Med. Biol., vol. 47, no. 21, pp. 3853-3863, Oct. 2002.

[11] Y. C. Shen, T. Lo, P. F. Taday, B. E. Cole, W. R. Tribe, and M. C. Kemp, "Detection and identification of explosives using terahertz pulsed spectroscopic imaging," Appl. Phys. Lett., vol. 86, no. 24, pp. 241116-1-2411163, Jun. 2005.

[12] K. Kawase, Y. Ogawa, Y. Watanabe, and H. Inoue, "Non-destructive terahertz imaging of illicit drugs using spectral fingerprints," Opt. Exp., vol. 11, no. 20, pp. 2549-2554, Oct. 2003.

[13] J. B. Jackson, J. Bowen, G. Walker, J. Labaune, G. Mourou, M. Menu, and K. Fukunaga, "A survey of terahertz applications in cultural heritage conservation science," IEEE Trans. Terahertz Sci. Technol., vol. 1, no. 1, pp. 220-231, Sep. 2011.

[14] G. T. Herman, Fundamentals of Computerized Tomography. New York: Academic, 1980.

[15] B. Recur, A. Younus, S. Salort, P. Mounaix, B. Chassagne, P. Desbarats, J-P. Caumes, and E. Abraham, "Investigation on reconstruction methods applied to 3D terahertz computed tomography," Opt. Exp., vol. 19, no. 6, pp. 5105-5117, Mar. 2011.

[16] A. Brahm, M. Kunz, S. Riehemann, G. Notni, and A. Tünnermann, "Volumetric spectral analysis of materials using terahertz-tomography techniques," Appl. Phys. B, vol. 100, no. 1, pp. 151-158, Mar. 2010.

[17] J. Xu and X.-C. Zhang, "Circular involute stage," Opt. Lett., vol. 29, no. 17, pp. 2082-2084, Sep. 2004.

[18] T. Yasui, E. Saneyoshi, and T. Araki, "Asynchronous optical sampling terahertz time-domain spectroscopy for ultrahigh spectral resolution and rapid data acquisition," Appl. Phys. Lett., vol. 87, no. 6, pp. 061101-1061101-3, Jun. 2005.

[19] A. Bartels, R. Cerna, C. Kistner, A. Thoma, F. Hudert, C. Janke, and T. Dekorsy, "Ultrafast time-domain spectroscopy based on high-speed asynchronous optical sampling," Rev. Sci. Instrum., vol. 78, no. 3, pp. 035107-1-035107-3, Mar. 2007.

[20] T. Yasui, M. Nose, A. Ihara, K. Kawamoto, S. Yokoyama, H. Inaba, K. Minoshima, and T. Araki, "Fiber-based, hybrid terahertz spectrometer using dual fiber combs," Opt. Lett., vol. 35, no. 10, pp. 1689-1691, May 2010.

[21] T. Yasui, Y. Kabetani, Y. Ohgi, S. Yokoyama, and T. Araki, "Absolute distance measurement of optically rough objects using asynchronousoptical-sampling terahertz impulse ranging," Appl. Opt., vol. 49, no. 28, pp. 5262-5270, Oct. 2010.

[22] Y. Kim and D.-S. Yee, "High-speed terahertz time-domain spectroscopy based on electronically controlled optical sampling," Opt. Lett., vol. 35, no. 22, pp. 3715-3717, Nov. 2010.

[23] Q. Wu, T. D. Hewitt, and X.-C. Zhang, "Two-dimensional electro-optic imaging of THz beams," Appl. Phys. Lett., vol. 69, no. 8, pp. 1026-1028, Aug. 1996.

[24] Z. Jiang and X.-C. Zhang, "Electro-optic measurement of THz field pulses with a chirped optical beam," Appl. Phys. Lett., vol. 72, no. 16, pp. 19451947, Apr. 1998.

[25] Z. Jiang, F. G. Sun, and X.-C. Zhang, "Terahertz pulse measurement with an optical streak camera," Opt. Lett., vol. 24, no. 17, pp. 1245-1247, Sep. 1999.

[26] J. Shan, A. S. Weling, E. Knoesel, L. Bartels, M. Bonn, A. Nahata, G. A. Reider, and T. F. Heinz, "Single-shot measurement of terahertz electromagnetic pulses by use of electro-optic sampling," Opt. Lett., vol. 25, no. 6, pp. 426-428, Mar. 2000 .

[27] Z. Jiang and X.-C. Zhang, "Single-shot spatiotemporal terahertz field imaging," Opt. Lett., vol. 23, no. 14, pp. 1114-1116, Jul. 1998.

[28] F. G. Sun, Z. Jiang, and X.-C. Zhang, "Analysis of terahertz pulse measurement with a chirped probe beam," Appl. Phys. Lett., vol. 73, no. 16, pp. 2233-2235, Oct. 1998.

[29] T. Yasuda, T. Yasui, T. Araki, and E. Abraham, "Real-time twodimensional terahertz tomography of moving objects," Opt. Commun., vol. 267, no. 1, pp. 128-136, Jun. 2006.

[30] T. Yasui, K. Sawanaka, A. Ihara, E. Abraham, M. Hashimoto, and T. Araki, "Real-time terahertz color scanner for moving objects," Opt. Exp., vol. 16, no. 2, pp. 1208-1221, Jan. 2008.

[31] M. Schirmer, M. Fujio, M. Minami, J. Miura, T. Araki, and T. Yasui, "Biomedical applications of a real-time terahertz color scanner," Biomed. Opt. Exp., vol. 1, no. 2, pp. 354-366, Sep. 2010.

[32] E. Abraham, Y. Ohgi, M. Minami, M. Jewariya, M. Nagai, T. Araki, and T. Yasui, "Real-time line projection for fast terahertz spectral computed tomography," Opt. Lett., vol. 36, no. 11, pp. 2119-2121, Jun. 2011.
[33] S. P. Jamison, J. Shen, A. M. MacLeod, W. A. Gillespie, and D. A. Jaroszynski, "High-temporal-resolution, single-shot characterization of terahertz pulses," Opt. Lett., vol. 28, no. 18, pp. 1710-1712, Sep. 2003.

[34] Z. Jiang, F. G. Sun, Q. Chen, and X.-C. Zhang, "Electro-optic sampling near zero optical transmission point," Appl. Phys. Lett., vol. 74, no. 9, pp. 1191-1193, Jan. 1999.

[35] F. Miyamaru, T. Yonera, M. Tani, and M. Hangyo, "Terahertz twodimensional electrooptic sampling using high speed complementary metal-oxide semiconductor camera," Jpn. J. Appl. Phys., vol. 43, no. 4A, pp. L489-L491, Mar. 2004.

[36] A. G. Stepanov, J. Hebling, and J. Kuhl, "Efficient generation of subpicosecond terahertz radiation by phase-matched optical rectification using ultrashort laser pulses with tilted pulse fronts," Appl. Phys. Lett., vol. 83, no. 15 , pp. 3000-3002, Aug. 2003.

[37] M. Jewariya, M. Nagai, and K. Tanaka, "Enhancement of terahertz wave generation by cascaded $\chi^{(2)}$ processes in $\mathrm{LiNbO}_{3}, "$ J. Opt. Soc. Amer. $B$, vol. 26, no. 9, pp. A101-A106, Sep. 2009.

[38] Z. Jiang and X.-C. Zhang, "Terahertz imaging via electrooptic effect," IEEE Trans. Microw. Theory Tech., vol. 47, no. 12, pp. 2644-2650, Dec. 1999

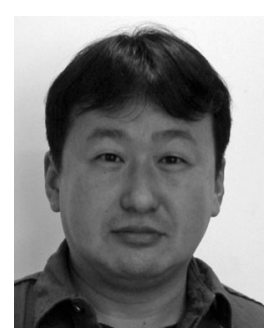

Takeshi Yasui received the Ph.D. degree in mechanical engineering from the University of Tokushima, Tokushima, Japan, in 1997.

From 1997 to 1999, he was a Postdoctoral Research Fellow in the National Research Laboratory of Metrology. He was with the Graduate School of Engineering Science, Osaka University, from 1999 to 2010 , and was briefly with the University of Bordeaux I in 2007 and the University of Littoral Côte d'Opale in 2010 as an Invited Professor. He is currently a Professor at the Institute of Technology and Science, The University of Tokushima, and an Invited Professor in the Graduate School of Engineering Science, Osaka University, Toyonaka, Japan. His research interests include terahertz instrumentation and metrology, laser stabilization, nonlinear optical microscopy, and ultrafast time-resolved measurement.

Dr. Yasui is a member of the Optical Society of America, the Japan Society of Applied Physics, the Optical Society of Japan, the Laser Society of Japan, the Japan Society of Medical Electronics and Biological Engineering, the Japan Society of Mechanical Engineers, and the Institute of Electronics, Information and Communication Engineers. He received the Award for the Most Promising Young Scientist from the Optical Society of Japan in 1998, the Sakamoto Award from the Japan Society of Medical Electronics and Biological Engineering in 2006, the Optics Paper Award from the Japan Society of Applied Physics, and the Funai Award from the Japan Society of Mechanical Engineers in 2009.

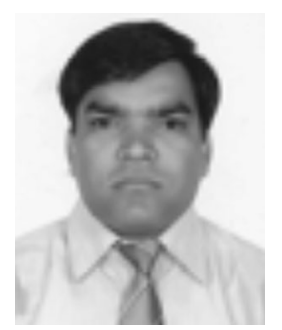

Mukesh Jewariya received the M.Sc. degree in physics from Indian Institute of Technology, Roorkee, India, in 2003, the M.Eng. degree in laser technology from Devi Ahilya Vishwavidyalaya, Indore, India, in 2006, and the D.Sc. degree in physics from Kyoto University, Kyoto, Japan, in 2010.

From 2011 to 2012, he was a Specially Appointed Researcher in the Renovation Center of Instruments for Science Education and Technology, Osaka University. He is currently an Assistant Professor at the Institute of Technology and Science, The University of Tokushima, Tokushima, Japan. His research interest includes generation of high-power terahertz pulse, terahertz imaging, and terahertz frequency metrology.

Dr. Jewariya is a member of the Japan Society of Applied Physics, the Physical Society of Japan, and the Optical Society of America. He is also Life Member of Indian Laser Association and Indian Physics Association.

Takashi Yasuda received the B.S. and M.S. degrees in mechanical engineering from Osaka University, Toyonaka, Japan, in 2001 and 2003, respectively.

He has been with the Hamamatsu Photonics K.K., Hamamatsu, Japan, since 2003. His research interests include terahertz imaging and spectroscopy.

Mr. Yasuda is a member of the Japan Society of Applied Physics. 
Markus Schirmer received the M.S. degree in physics from RWTH Aachen University, Aachen, Germany in 2011.

In 2009, he worked on the terahertz imaging as an exchange student in the Graduate School of Engineering Science, Osaka University, Toyonaka, Japan. He was employed as a Research Assistant at RWTH Aachen University, Germany, in 2011 after his graduation.

Mr. Schirmer is a member of the Deutsche Physikalische Gesellschaft.

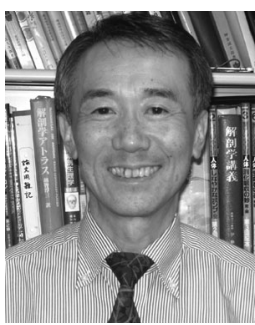

Tsutomu Araki received the Ph.D. degree in applied physics from Osaka University, Suita, Japan, in 1977, and the Ph.D. degree in medical sciences from the University of Tokushima, Tokushima, Japan, in 1986.

From 1978 to 1979 , he was a Postdoctoral Fellow in the University of Wisconsin. He was with the Faculty of Medicine of the University of Tokushima from 1979 to 1987 and the Faculty of Engineering of the University of Tokushima from 1987 to 1996. $\mathrm{He}$ is currently a Professor in the Graduate School of Engineering Science, Osaka University, Toyonaka, Japan. His research interests include development of a new biomedical and biophysical measurement system using an ultrafast pulse laser.

Dr. Araki is a member of the Society for Applied Spectroscopy, the Optical Society of America, the Japan Society of Mechanical Engineers, and the Japan Society of Applied Physics. He received the Sakamoto Award from the Japan Society of Medical Electronics and Biological Engineering, the Achievement Award from the Bioengineering Division in the Japan Society of Mechanical Engineers in 2006, the Achievement Award of Education and Research from the Osaka University in 2008, and the Funai Award from the Japan Society of Mechanical Engineers in 2009.

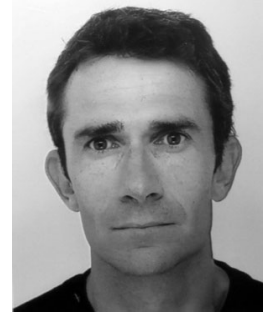

Emmanuel Abraham received the Ph.D. degree in physics from Bordeaux 1 University, Talence Cedex, France, in 1997.

In 1997-1998, he joined as a Postdoctoral Research Fellow the National Research Laboratory of Metrology, AIST, Tsukuba, Japan, and the FemtoSecond Technology project to study industrial applications of femtosecond lasers, including ultrafast optical Kerr gate. Since 1998, he has been an Assistant Professor at Bordeaux 1 University, working at the Laboratoire Ondes et Matière d'Aquitaine. He was invited in Osaka University as an Invited Researcher in 2007 and University of Tokushima as a Short-Term Invited Fellow Researcher of Japan Society for the Promotion of Science in 2010. His research interests include femtosecond time-resolved spectroscopy and nonlinear optics (ultrasfast electron transfer in organic molecules, energy transfer, elementary chemical reactions such as photoisomerization, etc.), applications of femtosecond infrared lasers to medical imaging (optical echography, propagation of optical pulses in turbid media). More recently, he has investigated the potential of terahertz radiation for the analysis of objects related to cultural heritage such as paintings, sculptures, ceramics, etc., by using pulsed and continuous millimeter-wave sources for 2-D imaging and 3-D terahertz computed tomography. 